\title{
Robótica Educativa na aprendizagem de Lógica de Programação: Aplicação e análise.
}

\author{
Eduardo Cambruzzi $^{1 *}$, Rosemberg Mendes de Souza ${ }^{1}$ \\ ${ }^{1}$ Instituto Federal de Educação, Ciência e Tecnologia da Bahia - IFBA \\ Valença - Bahia - Brasil \\ ec@ifba.edu.br, rosembergmendes.s@gmail.com
}

\begin{abstract}
In last decades the use of Educational Robotics as programming logic teaching tool has grown in schools and universities. While the interest and the number of projects increases in the academic, few are the studies to evaluate its effectiveness as object mediator to the learning. In this article, we build, applies and evaluating learning objects based on Educational Robotics as programming logic teaching tool. The results allow analyze the contributions that the Educational Robotics can bring to the learning of this discipline.
\end{abstract}

Resumo. Nas últimas décadas, o uso da Robótica Educativa como ferramenta de ensino de lógica de programação tem crescido, tanto nas escolas como nas universidades. Ao mesmo tempo que o interesse e o número de projetos para utilização desta ferramenta aumenta no âmbito acadêmico, poucos são os estudos que avaliam sua eficácia como objeto mediador na relação ensino-aprendizagem. Neste artigo, constrói-se, aplica-se e avalia-se Objetos de Aprendizagem baseados na Robótica Educativa como ferramenta de aprendizagem de Lógica de Programação. Os resultados obtidos permitem analisar as contribuições que a Robótica Educativa pode trazer para o aprendizado desta disciplina.

\section{Introdução}

A dificuldade em abstrair problemas do cotidiano e transformá-los em um conjunto de comandos sequenciais para serem executados por um computador, estão entre os grandes desafios que permeiam o desenvolvimento do chamado raciocínio lógico computacional [Ribeiro et al. 2011].

Entretanto, observa-se que alunos dos cursos de computação que não possuem ou que não desenvolvem a competência de abstração de problemas no início do curso, acabam por se desmotivar [Piva Jr and Freitas 2011].

A motivação dos alunos vem sendo apontada à décadas como um importante fator para melhoraria da relação ensino-aprendizagem e existem inúmeras ações que podem contribuir para o sucesso deste processo [Díaz Bordenave and Pereira 1991, Mattos 2001, Reis et al. 2015]. Uma destas ações é aproximar o abstrato e complexo, do concreto e do lúdico, integrando atividades com robôs programáveis ao cotidiano da sala de aula.

\footnotetext{
*Agradecemos ao Instituto Federal de Educação, Ciência e Tecnologia da Bahia - IFBA e a Fundação do Amparo à Pesquisa da Bahia - FAPESB.
} 
Muitos autores afirmam em seus estudos que, a Robótica Educativa é um importante instrumento para o desenvolvimento de habilidades que necessitam de pensamento lógico e abstrato. Um destes estudos foi realizado por [Ribeiro 2006] avalia os aspectos qualitativos da Robótica Educativa e aponta seus aspectos positivos para o aprimoramento do pensamento lógico. Para [Benitti et al. 2009], a Robótica Educativa serve de apoio ao ensino de Programação de computadores e os resultados obtidos pelo autor, indicam que os alunos apresentaram melhora na compreensão de conceitos computacionais abstratos e um aumento da motivação para realização das atividades propostas. Em outro estudo, [de Souza Pio et al. 2006], uma competição de robôs foi utilizada para estimular o interesse dos alunos e, em seus relatos, estes alunos dizem que passaram a compreender os erros que cometiam no processo de desenvolvimento do programa.

Neste artigo, descreve-se um estudo com aproximadamente cinquenta alunos durante dois anos, no qual, Objetos de Aprendizagem baseados na Robótica Educativa foram desenvolvidos e aplicados em uma turma do Ensino Técnico de Informática na disciplina de Lógica de Programação. Ao final, foram realizados testes quantitativos e qualitativos para avaliar o desenvolvimento médio destes alunos em relação aos conteúdos abordados nesta disciplina.

\section{Ensino e Aprendizagem de Lógica de Programação}

São múltiplos os fatores que dificultam a construção de um pensamento lógico computacional e consequentemente o aprendizado de Lógica de Programação, dentre eles destacamse: a falta de motivação, pois o aluno acredita que a disciplina é um obstáculo difícil de ser superado; o ensino instrucionista, o qual não estabelece uma relação entre a teoria e a prática; a dificuldade de assimilação das abstrações e o desenvolvimento de raciocínio lógico matemático [Hinterholz 2009]. Todos estes fatores têm levado à desmotivação, reprovação e evasão de alunos dos cursos de computação.

Buscando alternativas metodológicas para o ensino de Programação, muitos autores têm apontado que o uso de ferramentas lúdicas, na forma de Objetos de Aprendizagem, podem transformar conceitos abstratos em ações materiais e são úteis para atrair a atenção de alunos de todas as áreas de conhecimento, em especial das ciências da computação. Segundo [Macedo et al. 2005], a utilização de Objetos de Aprendizagem como ferramenta auxiliar nos processos de aprendizagem possui as seguintes qualidades: a) tornam as tarefas prazerosas; b) são desafiadoras; c) possuem dimensão simbólica; d) não limitam as possibilidades; e) expressam-se do modo construtivo e relacional.

\subsection{Objetos de Aprendizagem}

Objetos de Aprendizagem (OAs), principalmente os computacionais, tornaram-se nas últimas décadas uma importante estratégia pedagógica para atrair a atenção dos alunos. Conceitualmente, qualquer material que pode ser utilizado ou referenciado durante o processo de ensino-aprendizagem pode ser classificado com objeto aprendizagem [Hodgins et al. 2002].

Estes objetos têm se mostrado uma importante ferramenta educacional, não apenas em disciplinas ligadas à computação, mas nas mais variadas áreas de conhecimento como, história, geografia, matemática, química, etc. O principal objetivo dos OAs é complementar o processo de construção do conhecimento, melhorando a relação ensinoaprendizagem e expandido a sala de aula para mais próximo do contexto do aluno. 
O uso destes objetos dentro e fora das salas de aula, tem colaborado para a melhoria do processo de ensino-aprendizagem, uma vez que estes tornam as aulas mais interativas e participativas, [dos Santos and Costa 2006]. Pode-se afirmar também que, a utilização dos objetos de aprendizagem estimula a criatividade e a imaginação, contribuindo para a independência do aluno [Benitti et al. 2009]. Além disso, os OAs favorecem o dinamismo das aulas e a assimilação dos conhecimentos por parte dos alunos de forma lúdica e muitas vezes imperceptível, assim como ocorre durante o brincar. Um exemplo disto ocorre quando estes objetos são baseado na Robótica Educativa, já que trata-se de um ambiente de aprendizagem no qual os alunos podem analisar, discutir, montar e programar, de forma lúdica e interativa.

\subsection{Robótica Educativa}

A robótica é uma área multidisciplinar que se vale dos conhecimentos de outras ciências, como Engenharia Mecânica, Engenharia Elétrica e Inteligência Artificial para criação de robôs. Em sua aplicação habitual, os robôs são utilizados em linhas de produção industrial, em tarefas complexas ou insalubres, onde repetem infinitamente e com milimétrica precisão, uma série de operações previamente programadas.

Por outro lado, a Robótica Educativa foi criada para uso doméstico ou escolar e tem como principal característica, um ambiente de aprendizagem no qual os alunos podem montar, programar e analisar o comportamento de um robô ou sistema robotizado. Isto promove a socialização e a autonomia no aprendizado, criando um ambiente que reúne ciência, tecnologia e trabalho manual.

Devido à grande flexibilidade de uso da Robótica Educativa, esta pode ser aplicada nos mais diversos ramos de conhecimento, permitindo que educadores explorem conceitos teóricos de difícil compreensão de forma lúdica e atrativa [Benitti et al. 2009]. A principal virtude da Robótica Educativa é exigir do aluno a organização de tarefas e pensamentos, desde o planejamento, até a montagem mecânica e a programação da lógica do robô. Com isto, a cada passo do projeto é necessário agregar conhecimentos múltiplos para solucionar problemas, elevando gradualmente a complexidade de pensamento e, concomitantemente, o grau de atração dos alunos na resolução do problema.

É a partir de todos estes aspectos relacionados a capacidade interdisciplinar, a ludicidade e ao fascínio tecnológico que a Robótica Educativa exerce sobre os alunos, que se pode perceber o potencial de sua utilização para o ensino de Lógica de Programação.

\section{Metodologia Utilizada}

Com o objetivo de avaliar o uso da Robótica Educativa na relação ensino-aprendizagem de Lógica de Programação, realizou-se um estudo ao longo dos últimos dois anos. Este estudo envolveu duas turmas do curso técnico de computação do Instituto Federal de Educação, Ciência e Tecnologia da Bahia-campus Valença. Estas turmas, tratadas doravante por turma $\mathrm{A}$ e Turma $\mathrm{B}$, são formadas respectivamente por vinte e três e vinte $\mathrm{e}$ quatro alunos, suas características de gênero e idade são semelhantes e ambas cursam a mesma disciplina, denominada Lógica de Programação I.

Para diferenciar o modo como foram ministrados os conteúdos programáticos da disciplina em cada uma das turmas, define-se neste artigo duas metodologias: Tradicional e Robótica Educativa. 
Na Metodologia Tradicional, o ensino de programação é pré-definido em um plano de curso dividido em etapas, cada etapa com um conjunto de avaliações práticas e teóricas. Nestas aulas são apresentados inicialmente os conceitos teóricos e, $\log$ o após, problemas que devem ser resolvidos através do uso de uma linguagem de programação. Este método de ensino é amplamente utilizado no ensino de disciplinas de programação, seja no nível técnico ou superior.

Já a Robótica Educativa é baseada em um processo construcionista [Papert 1994]. Neste, os alunos são protagonistas na ação de aprendizagem, envolvendo-se na definição das tarefas e na resolução de problemas, geralmente baseados em uma proposição lúdica relacionada à realidade de seu cotidiano. Assim, nas aulas iniciais são explanados os conceitos fundamentais da disciplina e sistematicamente devem ser oferecidos cenários de estudo, por exemplo, desenvolver robôs capazes de desviar de obstáculos ou identificar cores no ambiente. Esta metodologia permite a participação ativa dos alunos no processo de aprendizagem, sem considerá-los expectadores do conhecimento, valorizando assim, suas experiências, envolvendo-os na análise, discussão e busca de soluções para os problemas apresentados.

Definidas as metodologias, a metodologia Tradicional foi aplicada aos alunos da turma $\mathrm{A}$, enquanto a metodologia da Robótica Educativa foi aplicado à turma $\mathrm{B}$. O espaço no qual ocorreram as aulas foi o mesmo para ambas as turmas: um laboratório de informática equipando com vinte computadores dispostos em uma ampla sala climatizada e equipada com dispositivos multimídia de som e imagem.

Entretanto, os alunos da turma B foram divididos em grupos de quatro alunos e cada um destes grupos recebeu um kit de robôs da linha LEGO Mindstorms. Após um debate entre os alunos, houve consenso entre os grupos para que fossem construídos robôs com características semelhantes, neste caso, um veículo com quatro rodas, equipado com um sensor ultrassônico e um sensor de cores. Assim, problemas semelhantes poderiam ser resolvidos por todos os grupos, sem a necessidade de reconstruir o robô para cada nova tarefa. Após cinquenta horas/aula utilizando os robôs em diferentes atividades, ${ }^{1}$ estes alunos foram apresentados a linguagem formal de programação. Durante vinte e duas horas/aula, problemas envolvendo comandos simples, operadores lógicos e matemáticos, condicionais e laços foram discutidos e implementados por estes alunos.

Ao final da disciplina foram realizados testes idênticos com alunos da turma A e B. Os dados obtidos a partir dos testes foram tabulados e analisados em função dos testes práticos e dos grupos de questões. Estes testes foram divididos em duas categorias: um teste prático de cunho quantitativo e um questionário com viés qualitativo.

a) Teste Prático: composto por cinco questões à serem implementadas em uma linguagem de programação em um período de duas horas/aula. As questões apresentam problemas em ordem crescente de complexidade sendo a primeira, uma questão para somar dois números, a segunda o cálculo da média de três números, a terceira depende do uso condicionais simples em sua solução, na quarta o aluno precisa utilizar condicionais aninhados e na quinta, laços de repetição. Acredita-se que, com este conjunto de questões é possível obter uma visão geral dos conhecimentos adquiridos pelos alunos em função

\footnotetext{
${ }^{1}$ Uma descrição detalhada das atividades e Objetos de Aprendizagem utilizados, podem ser encontrados em [Cambruzzi and de Souza 2014]
} 
dos conteúdos abordados na disciplina de Lógica de Programação I.

b) Teste Qualitativo: Este teste é composto por doze questões e busca levantar junto aos alunos quais são suas opiniões e percepções sobre a metodologia adotada e conteúdos abordados ao longo do curso. Na análise qualitativa desta pesquisa, os objetos pesquisados foram à princípio reduzidos aos critérios do questionário. Entretanto, como em toda pesquisa qualitativa, vale ressaltar que as reflexões dos pesquisadores a respeito das observações em sala de aula, suas impressões e digressões, se tornam dados por si só e parte significativa da interpretação dos resultados.

Opta-se assim, por um estudo empírico com um viés de análise baseado em relações entre variáveis quantitativas e qualitativas. Pois, métodos quantitativos permitem avaliar a importância e a tendência de populações a partir de associações estatísticas. Já métodos qualitativos, que consideram aspectos subjetivos ao fenômeno da aprendizagem, são ferramentas importantes para a análise de aspectos psicológicos, sociais, históricos e ambientais, fundamentais no campo da Educação. Algumas destas interpretações e percepções são apresentadas na Seção 4.

\section{Resultados e Análise}

Nesta seção, a Figura 1 apresenta os resultados que indicam a percepção do aluno em função da metodologia adotada e seu posterior interesse em permanecer no curso. Já a Figura 2, busca traçar uma relação entre a percepção subjetiva que o aluno tem sobre seu aprendizado e sua efetividade na resolução de problemas.

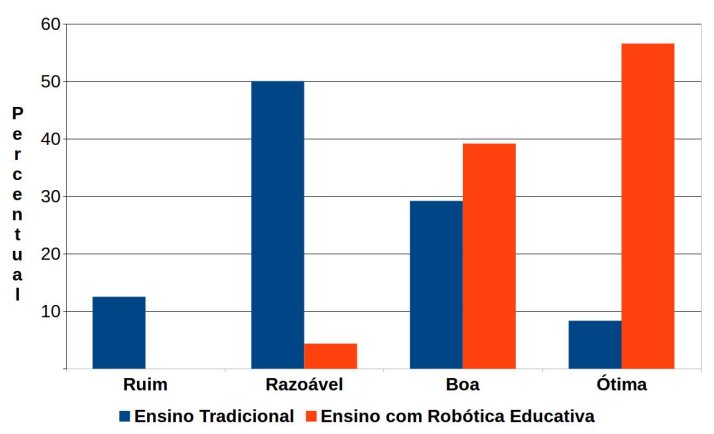

(a) Avaliação do aluno sobre a metodologia adotada.

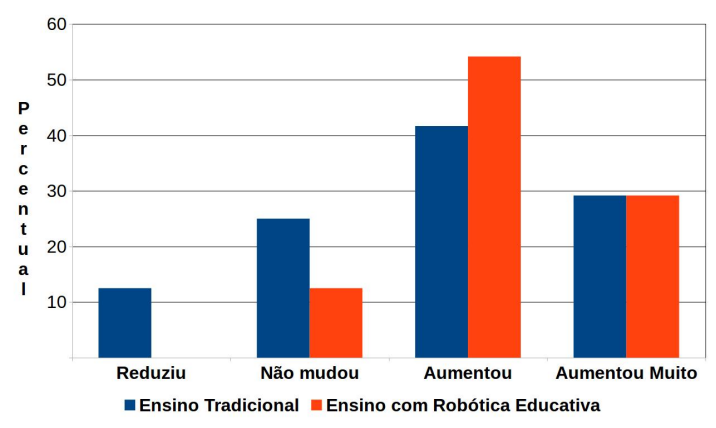

(b) Interesse do aluno pelo curso ao final da disciplina.

\section{Figura 1. Análise qualitativa da disciplina de Lógica de Programação}

Note na Figura 2(b) que, quando questionados sobre a metodologia de ensino adotada na disciplina, a Robótica Educativa tem notoriamente uma avaliação mais significativa que a metodologia Tradicional. Observa-se que 95\% dos alunos da turma B consideram a Robótica Educativa uma metodologia boa ou ótima enquanto apenas 37\% dos alunos da turma A, tem como boa ou ótima a metodologia Tradicional.

Entretanto, percebe-se na Figura 1(b), que a adoção de uma ou de outra metodologia não afeta significativamente a fixação do aluno no curso de computação. Observe que, apesar apenas $37 \%$ dos alunos submetidos a metodologia tradicional (turma A) a considerarem boa ou ótima $70 \%$ deles mantém seu interesse pelo curso após cursar a disciplina. 
Por outro lado, $95 \%$ dos alunos da turma Robótica (turma B)considerarem a metodologia boa ou ótima e $83 \%$ deles indica que a metodologia adotada contribuiu para aumentar seu interesse pelo curso. Isto indica uma diferença de aproximadamente $13 \%$ na fixação de alunos no curso de computação, apenas com a alteração da metodologia de ensino em uma única disciplina. Assim, é possível apontar como um fator gerador de interesse e de fixação de alunos no curso de computação, o uso de dispositivos lúdicos, como a Robótica Educativa.

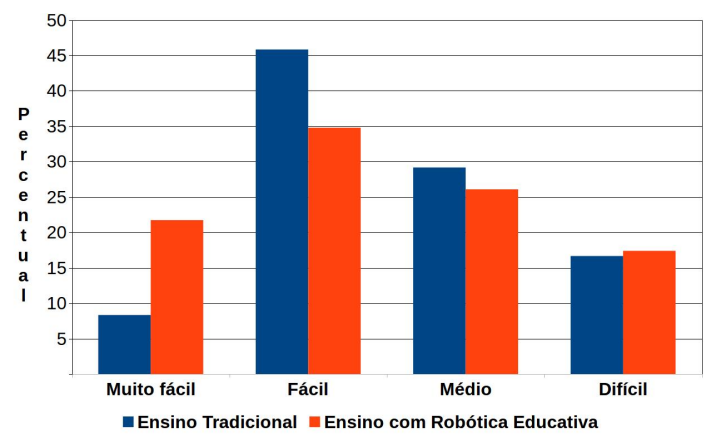

(a) Percepção do aluno em relação ao seu aprendizado.

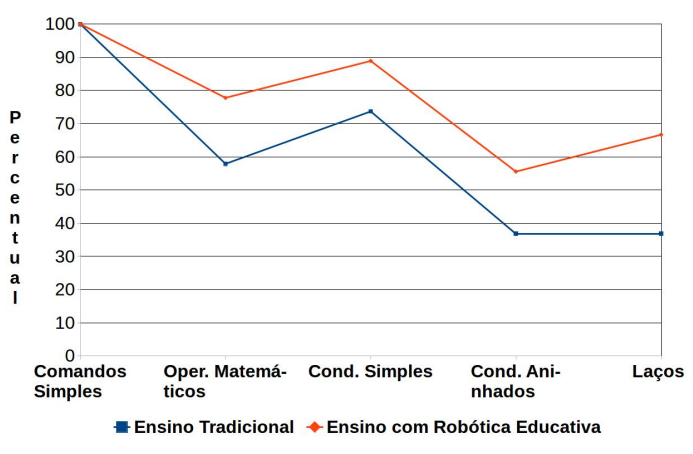

(b) Desempenho dos alunos no teste prático.

Figura 2. Análise quantitativa da disciplina de Lógica de Programação

Já a Figura 2 apresenta resultados conflitantes entre a percepção subjetiva dos alunos em relação ao seu aprendizado e seus resultados nos testes práticos. Note na Figura 2(a) que, aproximadamente 55\% dos alunos da turma A e 56\% do alunos da turma B, afirmam que os conteúdos abordados na disciplina de Lógica de Programação I são fáceis ou muito fáceis. Cabe ressaltar que os dados referentes a esta questão, foram coletados antes da realização dos testes práticos.

Observe na Figura2(b) o resultado do teste prático realizados com os alunos das turmas A e B. Apesar dos alunos de ambas as turmas indicarem que os assuntos tratados na disciplina foram fáceis ou muito fáceis, note como o desempenho da turma $\mathrm{B}$ foi significativamente melhor. Não apenas isto, à medida que a complexidade das tarefas aumentava, os alunos que utilizaram a Robótica Educativa foram mais efetivos em apresentar soluções corretas.

Enquanto na turma A que utilizou a metodologia Tradicional, 55\% dos alunos assumiram a disciplina fácil ou muito fácil, apenas $36 \%$ deles obtiveram exito na resolução de problemas mais complexos, que envolviam condicionais aninhados e laços de repetição. Entretanto, observe na mesma figura que os alunos da turma B, submetidos à Robótica Educativa, possuem uma percepção mais clara da complexidade da disciplina. Enquanto $56 \%$ da turma assume a disciplina como fácil ou muito fácil, mais de $60 \%$ destes alunos resolveram as questões mais complexas do teste prático. Isto indica uma coerência entre as habilidades adquiridas e a percepção da dificuldade de resolver problemas na turma submetida à Robótica Educativa. 


\section{Considerações Finais}

O ensino de Lógica de Programação depende do desenvolvimento de vários níveis de abstração por parte dos alunos. Os resultados obtidos neste estudo indicam que a utilização de uma metodologia mais lúdica e interativa como a da Robótica Educativa, colabora neste sentido. Isto pode ser observado pela significativa diferença de desempenho dos alunos expostos à metodologia da Robótica Educativa em relação aos que utilizaram a Metodologia Tradicional, principalmente na resolução de problemas mais complexos.

Também foram constatados bons resultados no que se refere a percepção dos estudantes quanto à utilização da Robótica Educativa como elemento motivador e facilitador da compreensão dos conceitos trabalhados nesta disciplina. Estes resultados podem ser observados principalmente nos altos índices de respostas positivas da turma B em relação a turma A, sobre a metodologia utilizada e em sua percepção de aprendizado.

Pode-se afirmar também que, a Robótica Educativa contribui significativamente em duas frentes: no aumento da aprendizagem dos alunos e sua percepção sobre os conhecimentos adquiridos e no interesse e fixação de alunos nos cursos de computação. Em estudos futuros pretende-se acompanhar estes alunos em disciplinas relacionadas à programação de computadores e avaliar se, sua formação inicial afeta seu desempenho nestas disciplinas.

\section{Referências}

Benitti, F. B. V., Vahldick, A., Urban, D. L., Krueger, M. L., and Halma, A. (2009). Experimentação com robótica educativa no ensino médio: ambiente, atividades e resultados. In XXVII Congresso da SBC-XV Workshop de Informática na Escola.

Cambruzzi, E. and de Souza, R. M. (2014). O uso da robótica educacional para o ensino de algoritmos. In Anais do V Encontro Anual de Tecnologia da Informação.

de Souza Pio, J. L., de Castro, T. H. C., and de Castro Júnior, A. N. (2006). A robótica móvel como instrumento de apoio à aprendizagem de computação. In XIII Simpósio Brasileiro de Informática na Educação, volume 1, pages 497-506.

Díaz Bordenave, J. and Pereira, A. M. (1991). Estratégias de ensino-aprendizagem. In Estratégias de ensino-aprendizagem. Vozes.

dos Santos, R. P. and Costa, H. A. X. (2006). Análise de metodologias e ambientes de ensino para algoritmos, estruturas de dados e programação aos iniciantes em computação e informática. volume 5 .

Hinterholz, O. (2009). Tepequém: uma nova ferramenta para o ensino de algoritmos nos cursos superiores em computação. In XVII-Anais do Workshop sobre Educação em Informática.

Hodgins, W., Duval, E., et al. (2002). Draft standard for learning object metadata. volume 1484 , pages $1-2002$.

Macedo, L., Petty, A. L. S., and PASSOS, N. C. (2005). Os jogos e o lúdico na aprendizagem escolar.

Mattos, M. M. (2001). Construção de abstrações em lógica de programação. In Anais do XXI Congresso da Sociedade Brasileira de Computação, Fortaleza. 
Papert, S. (1994). A máquina das crianças. Porto Alegre: Artmed.

Piva Jr, D. and Freitas, R. L. (2011). Estratégias para melhorar os processos de abstração na disciplina de algoritmos. In XXXI Congresso da Sociedade Brasileira de Computação, Natal/RN. Anais do XIX Workshop sobre Educação em Computação. Natal, RN: SBC, pages 1488-1497.

Reis, G. L., Souza, L. F. F., Barroso, M. F. S., Pereira, E. B., Nepomuceno, E. G., and Amaral, G. F. (2015). A relevância da integração entre universidades e escolas: um estudo de caso de atividades extensionistas em robótica educacional voltadas para rede pública de ensino. volume 2, pages 52-76.

Ribeiro, C. R. (2006). Robôcarochinha: um estudo qualitativo sobre a robótica educativa no $1^{\circ}$ ciclo do ensino básico.

Ribeiro, P. C., Martins, C. B., and Bernardini, F. C. (2011). A robótica como ferramenta de apoio ao ensino de disciplinas de programação ao em cursos de computação ao e engenharia. In XXII Simpósio Brasileiro de Informática na Educação. 